\title{
EMiR 2.0: A cognitive assistant robot for elderly
}

\author{
J. A. Rincon ${ }^{1}$, J. Palanca ${ }^{1}$, V. Botti ${ }^{1}$, A. Costa ${ }^{2}$, P. Novais ${ }^{2}$, V. Julian ${ }^{1}$, C. \\ Carrascosa $^{1}$ \\ 1 Universitat Politècnica de València. D. Sistemas Informáticos y Computación \\ \{jrincon, jpalanca, vbotti, vinglada, carrasco@dsic.upv.es\} \\ 2 Centro ALGORITMI, Escola de Engenharia, Universidade do Minho, Guimarães \\ \{acosta, pjon@di.uminho.pt\}
}

\begin{abstract}
This paper presents the EMiR robot, which is based on the RobElf robotic platform. EMiR has been developed as a cognitive assistant robot which is able to detect and to classify the emotional state of the human with whom it interacts. Moreover, EMiR integrates a powerful recommendation module that allows the robot to suggest activities to be done by the humans taking into account the emotional states among other aspects.
\end{abstract}

\section{Introduction}

In recent years we have seen an increase in assistant robots, which are offered as companions for the family and as assistants for the care of the elderly. These robots incorporate different image and audio processing techniques, which facilitate interaction with the human being. However, the information obtained through these techniques need to be processed using image and audio processing techniques in order to eliminate the existing noise. The result obtained is then analyzed using artificial intelligence (AI) tools, which using the processed data is able to identify people and / or objects, transform voice into text and text into voice facilitating the interaction between the human and the robot. Some of these robots have locomotion mechanisms that allow them to move within the environment, others are table robots which behave like personal assistants.

One of the characteristics that these robots currently possess is the ability to classify the emotional state of the human with whom they interact. As well as the ability to express an emotional state of their own, depending on the information they obtain from the environment around them. This characteristic, added to the previous ones, allows the users to feel a certain affinity for the robot, as well as, to facilitate the interaction with it.

This article presents EMiR, an assistant robot capable of interacting with humans, using voice and emotion detection as communication elements. Likewise, $E M i R$ uses powerful recommendation tools, which make it stand out from other robots in its class. These recommendation tools improve the way EMiR interacts with humans. 


\section{Related Work}

Recently, it is observable the increase of interest in assistive robots and their development. Current sociological shift demands technological solutions that are able to interact with elderly people. Thus, the sudden increase of the number of robots available that have as goal solving this sociological issue. Furthermore, they are increasingly refined and advanced in terms of aspect and features, being more human-like in both fields.

In recent years, we have seen a growing interest in robots, many of which are available to people. These robots have nice looks, a powerful background of artificial intelligence, navigation in complex environments, artificial vision, etc. Some of them, such as Pepper [1] and Romeo [2] from Aldebaran or Aido [3] or Buddy [3] from Frog Robotics, are presented as a new generation of home robots. One of the main applications of these robots is to assist older people in their daily life. Usually, these robots are used as a form of therapy or health care. In therapy field, we can find the Paro robot [4]. The Paro robot is an advanced interactive robot developed by AIST, which offers the well-known benefits of animal therapy to be administered to patients in environments such as hospitals. This robot was built to be a companion to children and elderly, prompting a positive emotional response due to its visual aspect. In the healthcare field we can find the Mabu robot of Catalia Health ${ }^{3}$, who learns over time about the personality, interests and therapeutic challenges of each patient. This allows Mabu to create conversations adapted to each patient. The structure of these conversations is based on well-known behavioral models of psychology to promote behavioral change.

From these projects we have observed that while the physical aspect, and its underlying impact (as displayed by PARO), is very advanced and the target of a careful development, they lack in terms of functionality. The actions that makes one human and their emotions are not explored by these projects and we believe that after the initial impact of the visual aspect it is what makes them really human-like. We, as humans, strive for human empathy, and is what our project aims for.

\section{$3 \quad \mathrm{EMiR}$}

$E M i R$ is based on the RobElf ${ }^{4}$ robotic platform. RobElf is a homemade robot focused mainly on accompanying and interacting with family members. RobElf is able to store and recognize people, tell stories for children and serve as a video surveillance tool. Although it is a good platform, RobElf does not have activity recommendation tools for family members. Although RobElf has the ability to smile and express emotions such as anger, sadness, happiness and surprise, he

\footnotetext{
${ }^{3}$ http://www.cataliahealth.com/introducing-the-mabu-personal-healthcarecompanion/

${ }^{4}$ https://www.robelf.com
} 
is not able to recognize them. Introducing these utilities would allow the robot a better way of interacting with family members. This would allow RobElf to integrate as one more member of the family.

However, the computational capacity of RobElf is very limited and the introduction of these two new utilities, it is necessary to use external services that can recommend and detect emotions. To detect emotions, EMiR uses deep learning as an artificial intelligence (AI) tool, allowing it to classify 7 emotions: afraid, angry, upset, happy, neutral, sad and surprised. EMiR uses this classification to recommend an activity that is according to the emotional state of the family member.

Fig. 1 shows the EMiR operating diagram, which has been divided into three steps. The first is the step of interaction with the human, this step involves the identification of the person, the interaction by voice, identifies the emotional state and interacts with the human with simple dialogues. Step second, EMiR modifies his facial expression to represent a series of possible emotional states (Fig. 2). In step third, EMiR uses the acquired information (person identification and emotional state) to suggest some type of activity. Finally, all the information obtained and the recommendations generated by the system are represented on an LCD screen.

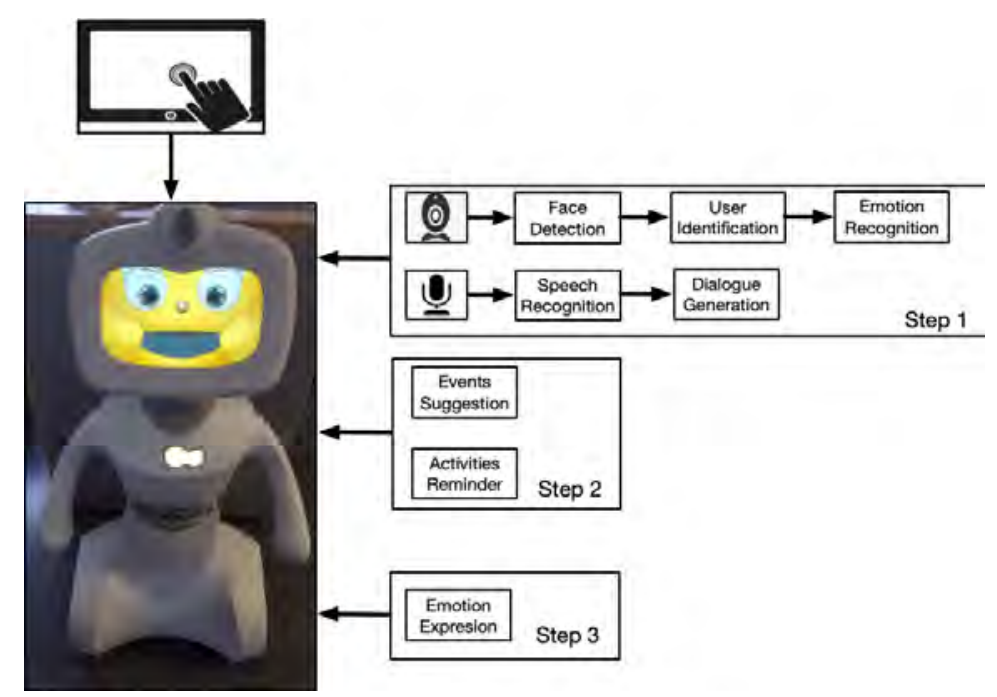

Fig. 1: Flow diagram of the EMiR robot operation. 


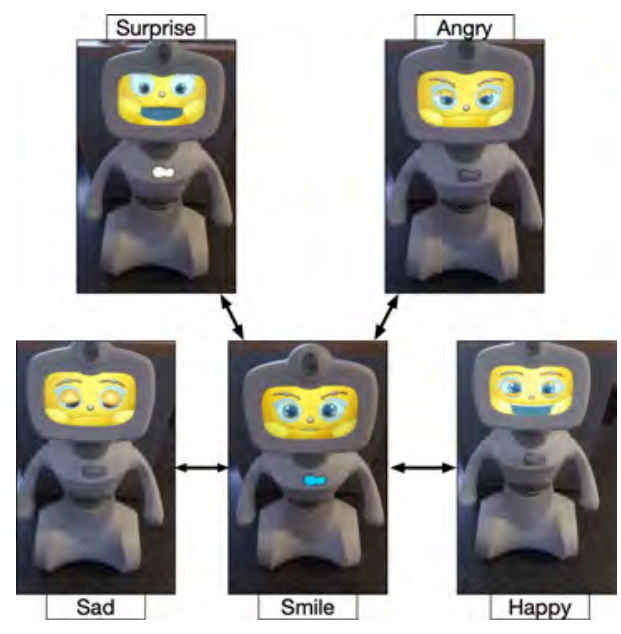

Fig. 2: Emotions expressed by EMiR.

\section{Conclusions and future work}

Robotics in the last decade have achieved a very important role in our society, which no longer perceives the robot as a tool used in industry. Robots are acquiring an important role in different scenarios and one of the most important is the assistance to elderly people. It is for this reason that in this article we have presented an approach of a commercial robot applied to the care of elderly people, to which we have added new capabilities for recognition of emotions and recommendation of activities.

\section{References}

1. Fumihide Tanaka, Kyosuke Isshiki, Fumiki Takahashi, Manabu Uekusa, Rumiko Sei, and Kaname Hayashi. Pepper learns together with children: Development of an educational application. IEEE-RAS International Conference on Humanoid Robots, 2015-December:270-275, 2015.

2. Giovanni Claudio, Fabien Spindler, and Francois Chaumette. Vision-based manipulation with the humanoid robot Romeo. In 2016 IEEE-RAS 16th International Conference on Humanoid Robots (Humanoids), pages 286-293. IEEE, nov 2016.

3. Ester Martinez-Martin and Angel P. del Pobil. Personal Robot Assistants for Elderly Care: An Overview, pages 77-91. Springer International Publishing, Cham, 2018.

4. K. Wada, T. Shibata, T. Saito, and K. Tanie. Effects of robot assisted activity to elderly people who stay at a health service facility for the aged. In $3 I E E E / R S J$ International Conference on Intelligent Robots and Systems (IROS), pages 28472852, 2003. 